\title{
A Comparison of Correlation Coefficients via a Three-Step Bootstrap Approach
}

\author{
Tahani A. Maturi (Corresponding author) \\ Department of Mathematical Sciences, University of Durham \\ Durham, DH1 3LE, England \\ E-mail: tahanistat@hotmail.com
}

Anga Elsayigh

Ain Shams University, Faculty of Commerce, Abbassia 11566, Cairo, Egypt

E-mail: angasoft@hotmail.com

\begin{abstract}
In this paper we compare ten correlation coefficients using a three-step bootstrap approach (TSB). A three-step bootstrap is applied to determine the optimal repetitions, $B$, to estimate the standard error of the statistic with certain degree of accuracy. The coefficients in question are Pearson product moment $(r)$, Spearman's rho $(\rho)$, Kendall's tau $(\tau)$, Spearman's Footrule $\left(F_{t}\right)$, Symmetric Footrule $(C)$, the Greatest deviation $\left(R_{g}\right)$, the Top - Down $\left(r_{T}\right)$, Weighted Kendall's tau $\left(\tau_{w}\right)$, Blest $(v)$, and Symmetric Blest's coefficient $\left(v^{*}\right)$. We consider a standard error criterion for our comparisons. However, since the rank correlation coefficients suffer from the tied problem that results from the bootstrap technique, we use existing modified formulae for some rank correlation coefficients, otherwise, the randomization tied-treatment is applied.
\end{abstract}

Keywords: A three-step bootstrap, Correlation coefficients, Pearson product moment, Spearman's rho, Kendall's tau, Spearman's Footrule, Symmetric Footrule, Greatest deviation, Top-Down, Weighted Kendall's tau, Blest, Symmetric Blest's coefficient

\section{Introduction}

One may be interested in the relationship between two factors or two variables and would wish to represent this relationship by a number or even using a statistical technique to make an inference. This number is called a correlation coefficient. The most common and well known correlation coefficient is the Pearson moment product coefficient. Some people may use this coefficient immediately ignoring the bivariate normality assumption of the data. Others may use a nonparametric rank correlation coefficient such as Spearman rho or Kendall tau for the same purpose. However the Pearson coefficient examines different aspects compared to Spearman and Kendall. Pearson coefficient considers the linearity of the relationship whereas Spearman and Kendall study the monotonicity of this relationship.

In some circumstances, we may have data with some outliers, in which case using the Greatest deviation correlation coefficient would be more suitable due to its robustness property against outliers. However, in other situations, assigning more emphasis on the top of the observations is required. For this purpose, several nonparametric coefficients are suggested, namely: the Top-Down, Weighted Kendall's tau, Blest, and Symmetric Blest's coefficient.

Usually the variance of the correlation coefficients is derived under the assumption that the null hypothesis that there is no correlation is true. Otherwise, it is intractable to calculate the variance without this assumption. Surely one would prefer using the correlation coefficient which produces small variation, and, consequently less standard error.

Borkowf (2000) presented a new nonparametric method for estimating the variance of Spearman's rho by calculating $\rho$ from a two-way contingency table with categories defined by the bivariate ranks. His method is a computer method depending on the data at hand like the bootstrap and jackknife methods. There are claims that his method is more accurate than the bootstrap and jackknife methods. However, it is more complicated and the differences in accuracy are small in comparison with other methods.

In this paper, we will apply the bootstrap method. Yet, the common issue is how many replications, $B$, should run to get the most accurate results required. One such approach is introduced by Andrews and Buchinsky $(2000 ; 2001 ; 2002)$ which is called a three-step approach. We will use this approach to determine the optimal number of replications for different degrees of accuracy. In addition we will use it for comparison, precisely, to estimate the standard error of the estimators, in our case the correlation coefficients, without imposing the null hypothesis. However, by using the bootstrap technique, the rank correlation coefficients suffer from tied problem. Therefore, we will use the existing adjusted formula for Spearman and Kendall coefficients (Hollander \& Wolfe, 1999); otherwise, the randomization tied-treatment is applied (Gibbons, 1971). 
Overviews of the correlation coefficients that are used in comparisons are given in Section 2. In Section 3, we will introduce some bootstrap notation and motivations. Then, in Section 4, we will summarize the three-step approach for estimating the standard error. The application of this approach on the correlation coefficients is introduced via the example in Section 5. Finally, conclusions are reported in the last section.

\section{Overview of the correlation coefficients}

Our data set consists of $n$ observations, where $\left(x_{i}, y_{i}\right), i=1,2, \ldots, n$ is a random sample from a continuous bivariate population, and let $p_{i}$ and $q_{i}$ be the ranks of $x_{i}$ and $y_{i}$, respectively. Here, we have ten correlation coefficients; Pearson moment product coefficient as a parametric correlation coefficient (Hollander \& Wolfe, 1999; Gibbons, 1971), five nonparametric correlation coefficients: Spearman's rho, Kendall's tau (Hollander \& Wolfe, 1999; Gibbons, 1971; Kendall, 1975), Spearman's Footrule or simply Footrule (Diaconis \& Graham, 1977; Franklin, 1988; Salama \& Quade, 2002; Ury \& Kleinecke, 1979), Symmetric Footrule (Salama et al., 2001), the Greatest deviation (Gideon \& Hollister, 1987), and four weighted nonparametric correlation coefficients: the Top-Down (Iman \& Conover, 1987), Weighted Kendall's tau (Shieh, 1998), Blest (Blest, 2000), and Symmetric Blest's coefficient (Genest \& Plante, 2003).

In Table 1 we summarize these coefficients which are involved in our comparisons. In the second and third columns their formulae are stated, yet the fourth column contains the adjusted formulae for Spearman and Kendall coefficients for when ties occur. For others coefficients where no adjusted formula is found in current literature, we will use the randomization tool (Gibbons, 1971) to deal with tied problem. The justification behind using the randomization method is that it behaves randomly as the bootstrap does. However, the most important property is that the randomization methods do not affect the null distribution of the rank correlation coefficient, so we do not need to adapt the null distribution for these coefficients.

\section{Bootstrap Motivation and Notation}

The bootstrap technique was first introduced by Efron in 1979 (Efron \& Tibshirani, 1994). It is a computer intensive method of statistical analysis that uses resampling to calculate standard errors, confidence intervals and significance tests. There are various applications of the bootstrap techniques in life sciences such as medical, social, and business science. They are applicable as a parametric or semi-parametric or nonparametric technique. In this paper we will consider the nonparametric bootstrap since the correlation coefficients (except Pearson) in question are nonparametric rank correlation coefficients.

Actually, the most common problem in bootstrap literature is choosing the optimal number of repetitions, $B$. By choosing different, small values it can result in different answers. By choosing extremely large values it gives a more accurate result, however more costly. Andrews and Buchinsky $(2000 ; 2001 ; 2002)$ introduced a three-step approach to determine the number of repetitions $B$ with pre-fixed degrees of accuracy which is applied for many different bootstrap problems, such as estimating the standard error, confidence interval, and p-value for classical statistical techniques.

The aim is to achieve the desired level of accuracy by choosing $B$. Accuracy is measured by the percentage deviation of the bootstrap standard error estimate, confidence interval length, test's critical value, test's p-value, or bias-corrected estimate based on $B$ bootstrap simulations from the corresponding ideal bootstrap quantities for which $B=\infty$. A bound of the relevant percentage deviation, $p d b$, is specified such as the actual percentage deviation is less than this bound with a specified probability, $(1-\delta)$ tends to one. That is, for given $(p d b, \delta)$, the optimal number of repetitions, $B^{*}$, satisfies

$$
P^{*}\left(100 \frac{\left|\hat{\lambda}_{B^{*}}-\hat{\lambda}_{\infty}\right|}{\hat{\lambda}_{\infty}} \leq p d b\right)=1-\delta,
$$

where $\lambda$ is a quantity of interest (the standard error in our case), $\hat{\lambda}_{\infty}$ is an "ideal" bootstrap estimate, and $\hat{\lambda}_{B}$ is the bootstrap approximation of $\hat{\lambda}_{\infty}$ based on $B$ bootstrap repetitions. Also, $P^{*}$ here represent the probability with respect to the randomness in the bootstrap samples.

In the rest of this section we will present the notation which we will use in the bootstrap framework. Our observed data is a sample of size $n: \mathbf{X}=\left(X_{1}, \ldots, X_{n}\right)^{\prime}$, where $X_{i}=\left(x_{i}, y_{i}\right) ; i=1,2, \ldots, n$. Let $\mathbf{X}^{*}=\left(X_{1}^{*}, \ldots, X_{n}^{*}\right)^{\prime}$ be a bootstrap sample of size $n$ based on the original sample $\mathbf{X}$. When the original sample $\mathbf{X}$ is comprised of independent and identically distributed (iid) or independent and nonidentically distributed (inid) random variables, the bootstrap sample $\mathbf{X}^{*}$ often is an iid sample of size $n$ drawn from some distribution $\hat{F}$. In this paper, $\hat{F}$ is the empirical distribution due to that we use the nonparametric bootstrap.

Let $\hat{\theta}=\hat{\theta}(\mathbf{X})$ be an estimator of a parameter $\theta_{0}$ based on the sample $\mathbf{X}$. Let $\hat{\theta}^{*}=\hat{\theta}\left(\mathbf{X}^{*}\right)$ denote the bootstrap estimator. Let $\left\{\mathbf{X}_{b}^{*}: b=1, \ldots, B\right\}$ denote $B$ iid bootstrap samples, each with the same distribution as $\mathbf{X}$. Let $\hat{\theta}_{b}^{*}=\hat{\theta}\left(\mathbf{X}_{b}^{*}\right)$, for $b=1, \ldots, B$, denote the corresponding $B$ bootstrap estimators.

\section{A three-step method for choosing the number of bootstrap repetitions $B$ for standard error estimates}

One of the application is to bootstrap standard error estimates for a scalar estimator $\hat{\theta}$, in our case $\hat{\theta}$ is a correlation coefficient. The quantities $\left(s e, \hat{s e}_{\infty}, \hat{s e}_{B}\right)$ in this case are the standard error, the "ideal" bootstrap standard error estimator, and 
the bootstrap standard error estimator based on $B$ bootstrap repetitions, respectively, where $E^{*}$ represent the expectation with respect to the randomness in the bootstrap samples. These quantities are given as

$$
\begin{gathered}
s e=\left[E[\hat{\theta}(X)-E(\hat{\theta}(X))]^{2}\right]^{1 / 2}, \\
\hat{s e_{\infty}}=\left[E^{*}\left[\hat{\theta}\left(X^{*}\right)-E^{*}\left(\hat{\theta}\left(X^{*}\right)\right)\right]^{2}\right]^{1 / 2}, \\
\hat{s e}_{B}=\left[\frac{1}{B-1} \sum_{b=1}^{B}\left(\hat{\theta}_{b}^{*}-\frac{1}{B} \sum_{c=1}^{B} \hat{\theta}_{c}^{*}\right)^{2}\right]^{1 / 2} .
\end{gathered}
$$

The three-step method, introduced by Andrews and Buchinsky (2000), depends on the estimation of the coefficient of excess kurtosis, $\gamma_{2}$, of the bootstrap distribution of the parameter estimator, where

$$
\gamma_{2}=\frac{E^{*}\left[\hat{\theta}\left(X^{*}\right)-E^{*}\left(\hat{\theta}\left(X^{*}\right)\right)\right]^{4}}{\hat{s e}_{\infty}^{4}}-3
$$

By using the three-step method we aim to choose a value of $B$ to achieve a desired accuracy of $\hat{s e}_{B}$ for estimating $\hat{s e}_{\infty}$ using pre-specified values of $(p d b, \delta)$. The method involves the following steps:

Step(1): Suppose $\gamma_{2}=0$, and a preliminary value of $B$, denoted $B_{0}$ where

$$
B_{0}=\operatorname{int}\left[\frac{5000 z_{1-\delta / 2}^{2}}{(p d b)^{2}}\right]
$$

and $\operatorname{int}(a)$ be the smallest integer greater than or equal to $a, z_{\alpha}$ represents the $\alpha$ quantile of the standard normal distribution.

Step(2): Simulate $B_{0}$ bootstrap, and compute

$$
\hat{\gamma}_{2 B_{0}}=\frac{\frac{1}{B_{0}-1} \sum_{b=1}^{B_{0}}\left(\hat{\theta}_{b}^{*}-\hat{\mu}_{B_{0}}\right)^{4}}{\left(\hat{s e}_{B_{0}}\right)^{4}}-3 \quad, \quad \hat{\mu}_{B_{0}}=\frac{1}{B_{0}} \sum_{b=1}^{B_{0}} \hat{\theta}_{b}^{*} .
$$

Simulate $R$, say $R=500$, bootstrap samples from $\left(\hat{\theta}_{1}^{*}, \ldots, \hat{\theta}_{B_{0}}^{*}\right)$, which can be written as $\Theta_{r}^{* *}=\left(\theta_{1 r}^{* *}, \ldots, \theta_{B_{0} r}^{* *}\right), r=1, \ldots, R$. Then compute

$$
\hat{\gamma}_{2 B_{0} R}=2 \hat{\gamma}_{2 B_{0}}-\frac{1}{R} \sum_{r=1}^{R} \hat{\gamma}_{2}\left(\Theta_{r}^{* *}\right)
$$

where

$$
\hat{\gamma}_{2}\left(\Theta_{r}^{* *}\right)=\frac{\frac{1}{B_{0}-1} \sum_{b=1}^{B_{0}}\left(\hat{\theta}_{r b}^{* *}-\frac{1}{B_{0}} \sum_{b=1}^{B_{0}} \hat{\theta}_{r b}^{* *}\right)^{4}}{\left[\frac{1}{B-1} \sum_{b=1}^{B}\left(\hat{\theta}_{r b}^{* *}-\frac{1}{B} \sum_{c=1}^{B} \hat{\theta}_{r c}^{* *}\right)^{2}\right]^{2}}-3 .
$$

Step(3): Let $B^{*}=\max \left(B_{0}, B_{1}\right)$, where

$$
B_{1}=\operatorname{int}\left[\frac{2500 z_{1-\delta / 2}^{2}\left(2+\hat{\gamma}_{2 B_{0} R}\right)}{(p d b)^{2}}\right]
$$

such that, if $\hat{\gamma}_{2 B_{0} R} \leq 0$ then the desired number of bootstrap repetitions is $B^{*}=B_{0}$, and if $\hat{\gamma}_{2 B_{0} R}>0$, compute $B_{1}-B_{0}$ additional bootstrap estimate $\left\{\hat{\theta}_{b}^{*}: b=B_{0}+1, \ldots, B_{1}\right\}$ before computing $\hat{s e}_{B^{*}}$.

\section{Example}

Let us consider the data from a study designed to ascertain the relative importance of the various factors contributing to tuna quality and to find objective methods for determining quality parameters and consumer preference (Hollander \& Wolfe, 1999). Table 2 gives values of the Hunter L measure of lightness $(X)$, along with panel scores $(Y)$ for nine lots of canned tuna (also see Figure 1).

The preliminary and the final values of the number of the replications for estimating the standard errors of our correlation coefficients are given in Tables 3 and 4. We will categorize our comparison in three blocks; a parametric correlation coefficient (Pearson), nonparametric rank correlation coefficients (Spearman, Kendall, Spearman's Footrule, Symmetric Footrule and the Greatest deviation coefficient). Finally, weighted nonparametric rank correlation coefficients (Top-Down, Weighted Kendall, Blest's coefficient and Symmetric Blest's coefficient). 
For example, let us focus on the situation when $p d b=5 \%$ and $\delta=0.01$, as in Table 5. Here the preliminary value of $B_{0}$ (for all correlation coefficients) is 1327 which is quite small as a starting point for any simulation, then the adjusted number of replications, $B_{1}$, is given in the third column in Table 5, which varies from one correlation coefficient to another. For example, for the Pearson correlation coefficient, this number, $B_{1}$ is more than double the preliminary value (increased by $177 \%$ ). However, for Footrule $B_{1}$ is just increased by $2.4 \%$ (from 1327 to 1527). For Kendall and Weighted Kendall, the number of replications, $B_{1}$, increased almost the same (between $38.9 \%$ and $39.41 \%$ ). Among the nonparametric correlation coefficients, Spearman has the highest increase in the number of replications, increased by $72.57 \%$.

According to the differences between the observed (calculated value from the data) and the bootstrap simulation estimate, we can see that for Kendall correlation coefficient, the difference between the actual value of the statistic (observed value) and the bootstrap estimate is small. However, the largest difference between the observed and the estimated values is for Weighted Kendall since we choose $m=5$ which means we ignore about a half of the data but this difference gap will reduce and is equal to that of Kendall at $m=n=9$. Also, the differences are quite similar for Pearson, Spearman, Footrule, and Greatest, which are all between 0.0312 and 0.0520 .

With respect to the standard error estimate, we found that the Pearson correlation coefficient has the smallest standard error, however, the Weighted Kendall has the largest value of standard error. In fact it is twice as much as the standard error of Pearson correlation coefficient. It is for the same reason that Weighted Kendall ignores some of the data (in our case 4 pairs of observations are ignored). In block 2, we found that the Greatest correlation coefficient has the smallest standard error which is expected since our data has some outliers (see Figure 1). However, Spearman and Footrule have large standard errors within this block.

In block 3, when our interest is focused on the initial (top) data, we noticed that the Symmetric Blest's coefficient has a smaller standard error than its asymmetric version and is also smaller than the other weighted correlation coefficients. However, as we mentioned above, Weighted Kendall has the largest standard error. Generally, the standard errors for correlation coefficients in block 3 are larger than those in block 1 and 2.

\section{Conclusion}

To conclude, one should use the Pearson correlation coefficient if the data meets the normality assumption, otherwise, the Greatest deviation performs well especially when the data has outliers. However, when we want emphasis on the initial (top) data, the Symmetric Blest's coefficient has lowest standard error amongst other weighted correlation coefficients.

\section{References}

Andrews D. W. K. and Buchinsky M. (2000). A three-step method for choosing the number of bootstrap repetitions. Econometrica, 68(1), 23-51.

Andrews D. W. K. and Buchinsky M. (2001). Evaluation of a three-step method for choosing the number of bootstrap repetitions. Journal of Econometrics, 103, 345-386.

Andrews D. W. K. and Buchinsky M. (2002). On the number of bootstrap repetitions for Bca confidence intervals. Econometric Theory, 18(4), 962-984.

Blest D. C. (2000). Rank correlation - An alternative measure. Australian \& New Zealand Journal of Statistics, 42(1), 101-111.

Borkowf C. B. (2000). A new nonparametric method for variance estimation and confidence interval construction for Spearman's rank correlation. Computational Statistics $\mathcal{E}$ Data Analysis, 34, 219-241.

Diaconis P. and Graham R. L. (1977). Spearman's Footrule as a measure of disarray. Journal of the Royal Statistical Society: Series B (Statistical Methodology), 39, 262-268.

Efron B. and Tibshirani R. J. (1994). An introduction to the bootstrap. Chapman \& Hall.

Franklin L. A. (1988). Exact tables of Spearman's Footrule for N=11(1)18 with estimate of convergence and errors for the normal approximation. Statistics $\mathcal{E}$ Probability Letters, 6, 399-406.

Genest C. and Plante J. (2003). On Blest's measure of rank correlation. The Canadian Journal of Statistics, 31(1), 1-18.

Gibbons J. D. (1971). Nonparametric statistical inference. McGRAW-HILL KOGAKUHA, LTD.

Gideon R. A. and Hollister R. A. (1987). A rank correlation coefficient resistant to outliers. Journal of the American Statistical Association: Theory and Methods, 82(398), 656-666.

Hollander M. and Wolfe D. A. (1999). Nonparametric statistical methods, 2nd edition. John Wiley \& Sons, INC.

Iman R. L. and Conover W. J. (1987). A measure of Top - Down correlation. Technometrics, 29(3), 351-357.

Kendall M. (1975). Rank correlation methods, 4th edition. Charles Griffin \& Company LTD. 
Salama I. A. and Quade D. (2002). Computing the distribution of Spearman's Footrule in $O\left(n^{4}\right)$ time. Journal of Statistical Computation and Simulation, 72(11), 895-898.

Salama I. A., Quade D. and Abdelfattah E. H. (2001). The symmetric Footrule. Communications in Statistics - Theory and Methods, 30(6), 1099-1109.

Shieh G. S. (1998). A weighted Kendall's tau statistic. Statistics \& Probability Letters, 39, 17-24.

Ury H. K. and Kleinecke D. C. (1979). Tables of the distribution of Spearman's Footrule. Applied Statistics, 28(3), 271-275.

Table 1. Overview of some correlation coefficients

\begin{tabular}{|c|c|c|c|}
\hline Corr.Coeff. & Formula & Definition & Modified formula for tied ranks \\
\hline Pearson & $r=\frac{\sum_{i=1}^{n}\left(x_{i}-\bar{x}\right)\left(y_{i}-\bar{y}\right)}{\sqrt{\sum_{i=1}^{n}\left(x_{i}-\bar{x}\right)^{2} \sum_{i=1}^{n}\left(y_{i}-\bar{y}\right)^{2}}}$ & & \\
\hline Spearman's rho & $\rho=1-\frac{6 D_{s}^{2}}{n\left(n^{2}-1\right)}$ & $D_{s}^{2}=\sum_{i=1}^{n}\left(p_{i}-q_{i}\right)^{2}$ & $\begin{array}{l}\rho=\frac{n\left(n^{2}-1\right)-6 D_{s}^{2}-\frac{1}{2}\left[\sum_{i=1}^{g} t_{i}\left(t_{i}^{2}-1\right)+\sum_{j=1}^{h} u_{j}\left(u_{j}^{2}-1\right)\right]}{\sqrt{\left[n\left(n^{2}-1\right)-\sum_{i=1}^{g} t_{i}\left(t_{i}^{2}-1\right)\right]\left[n\left(n^{2}-1\right)-\sum_{j=1}^{h} u_{i}\left(u_{i}^{2}-1\right)\right]}} \\
\text { where } g \text { denotes the number of tied } p \text { groups, } \\
t_{i} \text { is the size of tied } p \text { group } i, h \text { is the number } \\
\text { of tied } q \text { groups, and } u_{j} \text { is the size of tied } q \\
\text { group } j \text { (Hollander \& Wolfe, } 1999) .\end{array}$ \\
\hline Kendall's Tau & $\tau=\frac{2 K}{n(n-1)}$ & $\begin{array}{l}K=\sum_{i<j} \operatorname{sgn}\left(x_{i}-x_{j}\right) \operatorname{sgn}\left(y_{i}-y_{j}\right) \\
\text { where } \operatorname{sgn}(a)=1 \text { if } a>0 \\
\operatorname{sgn}(a)=0 \text { if } a=0 \text { and } \\
\operatorname{sgn}(a)=-1 \text { if } a<0\end{array}$ & $\begin{array}{l}\tau=\frac{2 K}{\sqrt{\left[n(n-1)-\sum_{i=1}^{g} t_{i}\left(t_{i}-1\right)\right]\left[n(n-1)-\sum_{j=1}^{h} u_{j}\left(u_{j}-1\right)\right]}} \\
\text { where } g \text { denotes the number of tied } X \text { groups, } \\
t_{i} \text { is the size of tied } X \text { group } i, h \text { is the number } \\
\text { of tied } Y \text { groups, and } u_{j} \text { is the size of the tied } \\
Y \text { group } j \text { (Hollander } \& \text { Wolfe, 1999). }\end{array}$ \\
\hline Spearmans Footrule & $F=\left\{\begin{array}{l}1-\frac{4 D}{n^{2}} \text { if } n \text { is even } \\
1-\frac{4 D}{n^{2}-1} \text { if } n \text { is odd }\end{array}\right.$ & $D(p, q)=\sum_{i=1}^{n}\left|q_{i}-p_{i}\right|$ & \\
\hline Symmetric Footrule & $C=\left\{\begin{array}{l}\frac{4 S}{n^{2}} \text { if } n \text { is even } \\
\frac{4 S}{n^{2}-1} \text { if } n \text { is odd }\end{array}\right.$ & $\begin{array}{l}S(p, q)=\frac{D\left(p, q^{*}\right)-D(p, q)}{2} \\
D\left(p, q^{*}\right)=\sum_{i=1}^{n}\left|p_{i}+q_{i}-(n+1)\right|\end{array}$ & \\
\hline Greatest deviation & $\begin{array}{l}R_{g}=\frac{d(\epsilon o q)-d(q)}{\left\lceil\frac{n}{2}\right\rceil} \\
\text { where }\lceil.\rceil \text { is the greatest } \\
\text { integer }\end{array}$ & $\begin{array}{l}d_{i}(q)=\sum_{j=1}^{i} I\left(q_{j}>i\right) \\
d(q)=\max \left[d_{i}(q)\right] \\
d_{i}(\epsilon \circ q)=\sum_{j=1}^{i} I\left(q_{j}<n+1-i\right) \\
d(\epsilon o q)=\max \left[d_{i}(\epsilon \circ q)\right]\end{array}$ & \\
\hline Top - Down & $r_{T}=\frac{\sum_{i=1}^{n} S_{p_{i}} S_{q_{i}}-n}{n-S_{1}}$ & $S_{i}=\sum_{j=i}^{n} \frac{1}{j}$ (Savage scores) & \\
\hline Weighted Kendalls & $\begin{array}{l}\tau_{w}=\frac{2 Q}{m(m-1)} \\
\text { where } m \text { is the number } \\
\text { of interesting rankings }\end{array}$ & $\begin{array}{l}Q=\sum_{1 \leq j<i \leq m} \operatorname{sgn}\left(q_{i}-q_{j}\right) \text { and } \\
p=\frac{m}{n}, m=\lceil(n+1) p\rceil, \text { where } \\
\lceil.\rceil \text { is the greatest integer }\end{array}$ & \\
\hline Blests Correlation & $v=\frac{2 n+1}{n-1}-\frac{12 w}{n(n+1)^{2}(n-1)}$ & $w=\sum_{i=1}^{n}(n-i+1)^{2} q_{i}$ & \\
\hline Symmetric Blest & $v^{*}=\frac{2 n+1}{n-1}-\frac{12\left(w_{1}+w_{2}\right)}{n(n+1)^{2}(n-1)}$ & $\begin{array}{l}w_{1}=\frac{1}{2} \sum_{i=1}^{n}(n+1-i)^{2} q_{i} \\
w_{2}=\frac{1}{2} \sum_{i=1}^{n}\left(n+1-q_{i}\right)^{2} i\end{array}$ & \\
\hline
\end{tabular}


Table 2. Tuna Lightness and Quality data set

\begin{tabular}{c|c|c}
\hline Lot & Hunter L value $(X)$ & Panel scores $(Y)$ \\
\hline 1 & 44.4 & 2.6 \\
2 & 45.9 & 3.1 \\
3 & 41.9 & 2.5 \\
4 & 53.3 & 5.0 \\
5 & 44.7 & 3.6 \\
6 & 44.1 & 4.0 \\
7 & 50.7 & 5.2 \\
8 & 45.2 & 2.8 \\
9 & 60.1 & 3.8 \\
\hline
\end{tabular}

Table 3. TSB results of estimating the standard errors of the correlation coefficients

\begin{tabular}{|c|c|c|c|c|c|c|c|c|}
\hline \multirow{3}{*}{$\begin{array}{l}\text { Pearson } \\
p d b(\%)\end{array}$} & \multicolumn{8}{|c|}{ Observed $(r)=0.5712$} \\
\hline & \multicolumn{4}{|c|}{$\delta=.05$} & \multicolumn{4}{|c|}{$\delta=.01$} \\
\hline & $B_{0}$ & $B_{1}$ & $\hat{r}$ & $s e_{B}$ & $B_{0}$ & $B_{1}$ & $\hat{r}$ & $s e_{B}$ \\
\hline 20 & 49 & 23 & 0.6621 & 0.2157 & 83 & 65 & 0.6365 & 0.2257 \\
\hline 15 & 86 & 68 & 0.6368 & 0.2238 & 148 & 977 & 0.6165 & 0.2185 \\
\hline 10 & 193 & 1180 & 0.6190 & 0.2193 & 332 & 1294 & 0.6188 & 0.2174 \\
\hline 5 & 769 & 1992 & 0.6160 & 0.2180 & 1327 & 3669 & 0.6232 & 0.2187 \\
\hline 1 & 19208 & 39025 & 0.6203 & 0.2186 & 33175 & 67616 & 0.6201 & 0.2193 \\
\hline \multirow[t]{2}{*}{ Spearman } & \multicolumn{8}{|c|}{ Observed $(\rho)=0.6000$} \\
\hline & \multicolumn{4}{|c|}{$\delta=.05$} & \multicolumn{4}{|c|}{$\delta=.01$} \\
\hline$p d b(\%)$ & $B_{0}$ & $B_{1}$ & $\hat{\rho}$ & $s e_{B}$ & $B_{0}$ & $B_{1}$ & $\hat{\rho}$ & $s e_{B}$ \\
\hline 20 & 49 & 56 & 0.5907 & 0.2727 & 83 & 83 & 0.5537 & 0.2867 \\
\hline 15 & 86 & 88 & 0.5398 & 0.3254 & 148 & 346 & 0.5285 & 0.3008 \\
\hline 10 & 193 & 420 & 0.5371 & 0.2965 & 332 & 570 & 0.5421 & 0.2895 \\
\hline 5 & 769 & 1207 & 0.5471 & 0.3026 & 1327 & 2290 & 0.5548 & 0.2938 \\
\hline 1 & 19208 & 29889 & 0.5569 & 0.2951 & 33175 & 51594 & 0.5555 & 0.2965 \\
\hline \multirow[t]{2}{*}{ Kendall } & \multicolumn{8}{|c|}{ Observed $(\tau)=0.4444$} \\
\hline & \multicolumn{4}{|c|}{$\delta=.05$} & \multicolumn{4}{|c|}{$\delta=.01$} \\
\hline$p d b(\%)$ & $B_{0}$ & $B_{1}$ & $\hat{\tau}$ & $s e_{B}$ & $B_{0}$ & $B_{1}$ & $\hat{\tau}$ & $s e_{B}$ \\
\hline 20 & 49 & 40 & 0.4659 & 0.2448 & 83 & 74 & 0.4473 & 0.2574 \\
\hline 15 & 86 & 75 & 0.4474 & 0.2564 & 148 & 336 & 0.4198 & 0.2726 \\
\hline 10 & 193 & 402 & 0.4235 & 0.2674 & 332 & 534 & 0.4310 & 0.2638 \\
\hline 5 & 769 & 1029 & 0.4345 & 0.2708 & 1327 & 1839 & 0.4401 & 0.2673 \\
\hline 1 & 19208 & 22832 & 0.4449 & 0.2667 & 33175 & 39455 & 0.4445 & 0.2661 \\
\hline \multirow[t]{2}{*}{ Footrule } & \multicolumn{8}{|c|}{ Observed $\left(F_{t}\right)=0.2000$} \\
\hline & \multicolumn{4}{|c|}{$\delta=.05$} & \multicolumn{4}{|c|}{$\delta=.01$} \\
\hline$p d b(\%)$ & $B_{0}$ & $B_{1}$ & $\hat{F}_{t}$ & $s e_{B}$ & $B_{0}$ & $B_{1}$ & $\hat{F}_{t}$ & $s e_{B}$ \\
\hline 20 & 49 & 43 & 0.1816 & 0.2970 & 83 & 65 & 0.1663 & 0.3209 \\
\hline 15 & 86 & 63 & 0.1581 & 0.3047 & 148 & 157 & 0.1166 & 0.3196 \\
\hline 10 & 193 & 190 & 0.1202 & 0.3265 & 332 & 340 & 0.1253 & 0.3109 \\
\hline 5 & 769 & 777 & 0.1344 & 0.3109 & 1327 & 1359 & 0.1527 & 0.3071 \\
\hline 1 & 19208 & 20843 & 0.1496 & 0.3115 & 33175 & 35778 & 0.1495 & 0.3131 \\
\hline Symmetric & \multicolumn{8}{|c|}{ Observed $(C)=0.4500$} \\
\hline Footrule & \multicolumn{4}{|c|}{$\delta=.05$} & \multicolumn{4}{|c|}{$\delta=.01$} \\
\hline$p d b(\%)$ & $B_{0}$ & $B_{1}$ & $\hat{C}$ & $s e_{B}$ & $B_{0}$ & $B_{1}$ & $\hat{C}$ & $s e_{B}$ \\
\hline 20 & 49 & 49 & 0.4643 & 0.2481 & 83 & 83 & 0.4578 & 0.2662 \\
\hline 15 & 86 & 82 & 0.4570 & 0.2521 & 148 & 281 & 0.4461 & 0.2615 \\
\hline 10 & 193 & 321 & 0.4327 & 0.2571 & 332 & 507 & 0.4250 & 0.2726 \\
\hline 5 & 769 & 1083 & 0.4344 & 0.2611 & 1327 & 1772 & 0.4391 & 0.2577 \\
\hline 1 & 19208 & 26162 & 0.4363 & 0.2642 & 33175 & 44740 & 0.4363 & 0.2651 \\
\hline
\end{tabular}


Table 4. TSB results of estimating the standard errors of the correlation coefficients, contd.

\begin{tabular}{|c|c|c|c|c|c|c|c|c|}
\hline \multirow{3}{*}{$\begin{array}{l}\text { Greatest } \\
p d b(\%)\end{array}$} & \multicolumn{8}{|c|}{ Observed $\left(R_{g}\right)=0.2000$} \\
\hline & \multicolumn{4}{|c|}{$\delta=.05$} & \multicolumn{4}{|c|}{$\delta=.01$} \\
\hline & $B_{0}$ & $B_{1}$ & $\hat{R_{g}}$ & $s e_{B}$ & $B_{0}$ & $B_{1}$ & $\hat{R_{g}}$ & $s e_{B}$ \\
\hline 20 & 49 & 51 & 0.2706 & 0.1952 & 83 & 104 & 0.2596 & 0.2444 \\
\hline 15 & 86 & 108 & 0.2630 & 0.2440 & 148 & 204 & 0.2235 & 0.2404 \\
\hline 10 & 193 & 259 & 0.2247 & 0.2434 & 332 & 390 & 0.2369 & 0.2345 \\
\hline 5 & 769 & 790 & 0.2311 & 0.2348 & 1327 & 1444 & 0.2312 & 0.2351 \\
\hline 1 & 19208 & 22685 & 0.2311 & 0.2297 & 33175 & 37942 & 0.2301 & 0.2296 \\
\hline \multirow[t]{2}{*}{ Top-Down } & \multicolumn{8}{|c|}{ Observed $\left(r_{T}\right)=0.7392$} \\
\hline & \multicolumn{4}{|c|}{$\delta=.05$} & \multicolumn{4}{|c|}{$\delta=.01$} \\
\hline$p d b(\%)$ & $B_{0}$ & $B_{1}$ & $\hat{r_{T}}$ & $s e_{B}$ & $B_{0}$ & $B_{1}$ & $\hat{r_{T}}$ & $s e_{B}$ \\
\hline 20 & 49 & 38 & 0.6375 & 0.3793 & 83 & 61 & 0.6290 & 0.3615 \\
\hline 15 & 86 & 61 & 0.6247 & 0.3615 & 148 & 185 & 0.6169 & 0.3767 \\
\hline 10 & 193 & 260 & 0.6020 & 0.3770 & 332 & 367 & 0.5986 & 0.3714 \\
\hline 5 & 769 & 986 & 0.6281 & 0.3607 & 1327 & 1861 & 0.6329 & 0.3544 \\
\hline 1 & 19208 & 25664 & 0.6341 & 0.3537 & 33175 & 44175 & 0.6333 & 0.3548 \\
\hline Weighted & \multicolumn{8}{|c|}{ Observed $\left(\tau_{w}\right)=0.8000, m=5$} \\
\hline Kendall & \multicolumn{4}{|c|}{$\delta=.05$} & \multicolumn{4}{|c|}{$\delta=.01$} \\
\hline$p d b(\%)$ & $B_{0}$ & $B_{1}$ & $\hat{\tau_{w}}$ & $s e_{B}$ & $B_{0}$ & $B_{1}$ & $\hat{\tau_{w}}$ & $s e_{B}$ \\
\hline 20 & 49 & 44 & 0.4859 & 0.3721 & 83 & 65 & 0.4762 & 0.3702 \\
\hline 15 & 86 & 65 & 0.4720 & 0.3784 & 148 & 175 & 0.4155 & 0.4304 \\
\hline 10 & 193 & 217 & 0.4132 & 0.4181 & 332 & 327 & 0.4160 & 0.4069 \\
\hline 5 & 769 & 968 & 0.4355 & 0.4209 & 1327 & 1850 & 0.4329 & 0.4150 \\
\hline 1 & 19208 & 26252 & 0.4394 & 0.4200 & 33175 & 45077 & 0.4402 & 0.4189 \\
\hline \multirow[t]{2}{*}{ Blest } & \multicolumn{8}{|c|}{ Observed $(v)=0.6000$} \\
\hline & \multicolumn{4}{|c|}{$\delta=.05$} & \multicolumn{4}{|c|}{$\delta=.01$} \\
\hline$p d b(\%)$ & $B_{0}$ & $B_{1}$ & $\hat{v}$ & $s e_{B}$ & $B_{0}$ & $B_{1}$ & $\hat{v}$ & $s e_{B}$ \\
\hline 20 & 49 & 40 & 0.6012 & 0.2655 & 83 & 74 & 0.5808 & 0.2818 \\
\hline 15 & 86 & 71 & 0.5825 & 0.2776 & 148 & 344 & 0.5722 & 0.3009 \\
\hline 10 & 193 & 344 & 0.5492 & 0.3151 & 332 & 648 & 0.5503 & 0.3153 \\
\hline 5 & 769 & 1247 & 0.5400 & 0.3171 & 1327 & 1994 & 0.5313 & 0.3169 \\
\hline 1 & 19208 & 27959 & 0.5391 & 0.3154 & 33175 & 49867 & 0.5381 & 0.3150 \\
\hline Symmetric & \multicolumn{8}{|c|}{ Observed $\left(v^{*}\right)=0.6250$} \\
\hline Blest & \multicolumn{4}{|c|}{$\delta=.05$} & \multicolumn{4}{|c|}{$\delta=.01$} \\
\hline$p d b(\%)$ & $B_{0}$ & $B_{1}$ & $\hat{v}^{*}$ & $s e_{B}$ & $B_{0}$ & $B_{1}$ & $\hat{v}^{*}$ & $s e_{B}$ \\
\hline 20 & 49 & 37 & 0.6159 & 0.2492 & 83 & 68 & 0.5968 & 0.2645 \\
\hline 15 & 86 & 66 & 0.5981 & 0.2614 & 148 & 342 & 0.5855 & 0.2869 \\
\hline 10 & 193 & 347 & 0.5685 & 0.2981 & 332 & 667 & 0.5667 & 0.2980 \\
\hline 5 & 769 & 1246 & 0.5593 & 0.2987 & 1327 & 1975 & 0.5514 & 0.2973 \\
\hline 1 & 19208 & 28237 & 0.5586 & 0.2977 & 33175 & 50212 & 0.5575 & 0.2976 \\
\hline
\end{tabular}

Table 5. TSB results when $p d b=5 \%$ and $\delta=0.01$

\begin{tabular}{lcccccc}
\hline Corr.Coeff. & Observed $(O)$ & $B_{1}$ & $100 \frac{B_{1}-B_{0}}{B_{0}}$ & Estimate $(E)$ & $|O-E|$ & $s e_{B}$ \\
\hline Pearson & 0.5712 & 3669 & 176.49 & 0.6232 & 0.0520 & 0.2187 \\
\hline Spearman & 0.6000 & 2290 & 72.57 & 0.5548 & 0.0452 & 0.2938 \\
Kendall & 0.4444 & 1839 & 38.58 & 0.4401 & 0.0043 & 0.2673 \\
Footrule & 0.2000 & 1359 & 2.41 & 0.1527 & 0.0473 & 0.3071 \\
Symmetric footrule & 0.4500 & 1772 & 33.53 & 0.4391 & 0.0109 & 0.2577 \\
Greatest & 0.2000 & 1444 & 8.82 & 0.2312 & 0.0312 & 0.2351 \\
\hline Top-down & 0.7392 & 1861 & 40.24 & 0.6329 & 0.1063 & 0.3544 \\
Weighted Kendall & 0.8000 & 1850 & 39.41 & 0.4329 & 0.3671 & 0.4150 \\
Blest & 0.6000 & 1994 & 50.26 & 0.5313 & 0.0687 & 0.3169 \\
Symmetric Blest & 0.6250 & 1975 & 48.83 & 0.5514 & 0.0736 & 0.2973 \\
\hline
\end{tabular}




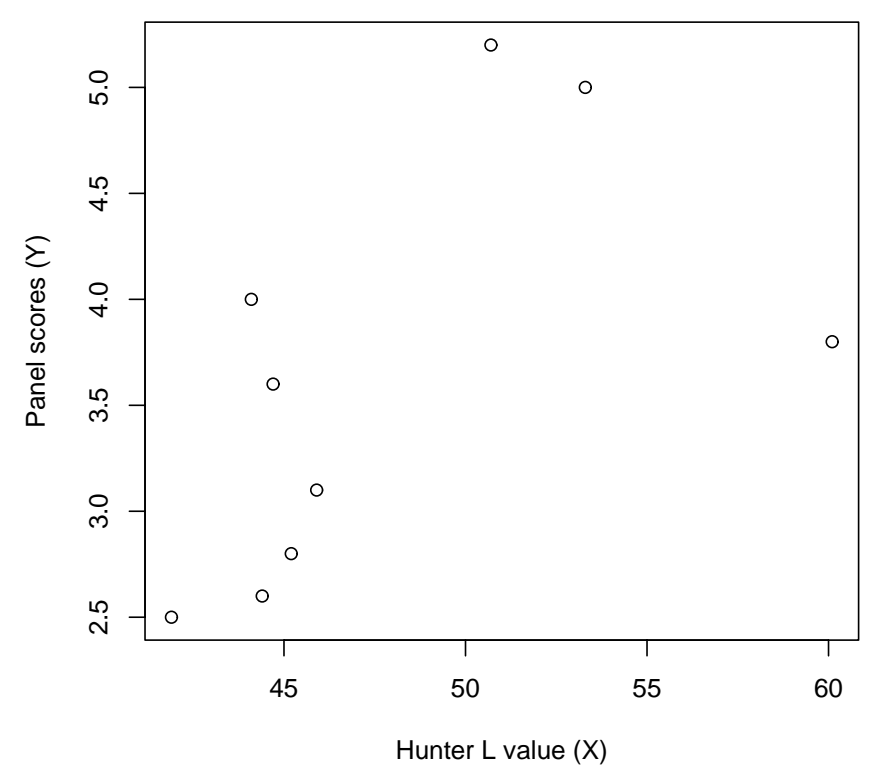

Figure 1. A simple scatter plot for Tuna Lightness and Quality data 